conditions. In the present paper he extends this method to double series, and discusses the application of the method to convergent double series. It is found that the summation functions of the familiar methods of Cesàro, Hölder, Borel, LeRoy, Riesz, Vallee-Poussin, etc., can be used in building up summation formulas for double series.

B. A. Bernstein, Secretary of the Section.

\title{
ON A CERTAIN GENERATION OF RATIONAL CIRCULAR AND ISOTROPIC CURVES.
}

BY PROFESSOR ARNOLD EMCH.

(Read before the American Mathematical Society December 28, 1917.)

\section{Introduction.}

A CIRCUlar curve contains the circular points at infinity, or the isotropic points of the plane, as single, or as singular points. A plane isotropic curve is defined as a curve, all of whose infinite points are absorbed by the isotropic points. The equation of such a curve, which is necessarily of even order, in cartesian coordinates may be written in the form

$$
\left(x^{2}+y^{2}\right)^{k}+\varphi(x, y)=0,
$$

in which $\varphi(x, y)$ is a polynomial of degree $2 k-1$ at most.

If $P(\xi, \eta)$ is a fixed point and $A(x, y)$ any other point so that $P A=\rho$, and $\theta$ the angle between $P A$ and the positive direction of the $x$-axis, then the coordinates of $A$ are $x=\xi+\rho \cos \theta, y=\eta+\rho \sin \theta$, and satisfy equation (1) when $A$ is on the curve. The condition for this is an equation of the form

$$
\rho^{2 k}+\alpha_{1} \rho^{2 k-1}+\alpha_{2} \rho^{2 k-2}+\cdots+\alpha_{2 k-1} \rho+\alpha_{2 k}=0,
$$

in which $\alpha_{1}, \alpha_{2}, \cdots, \alpha_{2 k-1}$ are coefficients, which, in general, depend on $\xi, \eta, \theta$ and the coefficients of (1); while $\alpha_{2 k}$ is independent of $\theta$. The roots $\rho_{1}, \rho_{2}, \cdots, \rho_{2 k}$ of (2) are the distances $P A_{i}(i=1,2,3, \cdots, 2 k)$ of the points of inter- 
section $A_{1}, A_{2}, \cdots, A_{2 k}$ of the transversal through $P$, including an angle $\theta$ with the positive part of the $x$-axis. Evidently the product

$$
\rho_{1} \rho_{2} \cdots \rho_{2 k}=\alpha_{2 k}
$$

is constant for all transversals through $P$.

This constant product is called the power of the point $P$ with respect to the isotropic curve. This is one of the principal properties* of an isotropic curve. In fact an isotropic curve may also be defined by this property. In this paper I shall establish the necessary and sufficient conditions for the form of parametric representation of rational circular, in particular of rational isotropic curves, and their generation by rational transformations in a complex plane. As an application the complete representation of all rational circular cubics, and all rational isotropic quartics will be given.

2. Parametric Representation of Rational Circular and Isotropic

Let

Curves.

$$
x=\frac{F(t)}{H(t)}, \quad y=\frac{G(t)}{H(t)},
$$

in which $F, G, H$ are polynomials in $t$, with real coefficients, without a common factor, represent a rational curve. To real values of $t$ correspond real values of $x$ and $y$, or real points on the curve. The curve passes once through each of the isotropic points, when for a complex value $t_{i}$ of $t$

$$
\text { (5) } \quad \lim _{t=t_{i}}(x)=\infty, \quad \lim _{t \doteq t_{i}}(y)=\infty, \quad \lim _{t=t_{i}}\left(\frac{y}{x}\right)=+i \text {, }
$$

and, as a consequence, also for the conjugate value $\bar{t}_{i}$

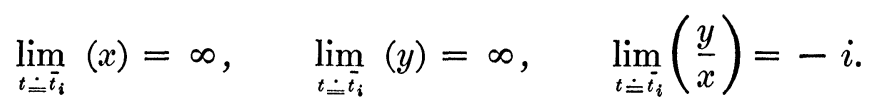

The algebraic sign of $i$ might, of course, be reversed. From this it follows that a necessary condition that the curve (4) pass through the isotropic points is that $H(t)=0$ has complex roots which are not common to $F(t)=0$ and $G(t)=0$. But

* E. Pascal, Repertorium der höhern Mathematik, vol. 2 (first half), pp. 436-438 (1910). 
complex roots of $H(t)=0$ occur in conjugate pairs. Let $t_{1}, \bar{t}_{1} ; t_{2}, \bar{t}_{2} ; \cdots ; t_{k}, \bar{t}_{k}$ be complex roots of this equation, so that $H(t)$ may be written in the form

$$
\begin{array}{r}
H(t)=\left[\left(t-t_{1}\right)\left(t-t_{2}\right) \cdots\left(t-t_{k}\right)\right]\left[\left(t-\bar{t}_{1}\right)\left(t-\bar{t}_{2}\right)\right. \\
\left.\cdots\left(t-\bar{t}_{k}\right)\right] \Phi(t),
\end{array}
$$

where $\Phi(t)$ is a polynomial in $t$, which contains none of the other factors of $H(t)$. The two brackets in (7) may be written in the form

and

$$
t^{k}+\alpha_{1} t^{k-1}+\alpha_{2} t^{k-2}+\cdots+\alpha_{k},
$$

$$
t^{k}+\bar{\alpha}_{1} t^{k-1}+\bar{\alpha}_{2} t^{k-2}+\cdots+\bar{\alpha}_{k},
$$

and are clearly conjugate expressions, so that the first may be written in the form $r-i s$, the second in the form $r+i s$, in which $r$ and $s$ are in general polynomials in $t$ of degree $k$. Hence, (7) has the form

$$
H(t)=\left(r^{2}+s^{2}\right) \Phi(t) .
$$

Incidentally we have proved

Theorem 1: If a polynomial equation with one unknown, and real coefficients, has only imaginary roots, then the polynomial may be represented as the sum of the squares of two other polynomials.

Suppose now that $t_{i}$ satisfies the equation $r-i s=0$, and that for this value of $t$ not both $r$ and $s$ vanish simultaneously. Then $t_{i}$, according to (5), defines an isotropic point of the curve, when $t_{i}$ is a root of the equation

$$
G(t)-i F(t)=0 \text {, }
$$

or

$$
F(t)+i G(t)=0 .
$$

Consequently, when the roots $t_{1}, t_{2}, \cdots, t_{k}$ of $r-i s=0$ all define isotropic points, (9) may be written in the form

$$
F(t)+i G(t)=(r-i s) \Psi(t),
$$

where $\Psi(t)$ is a polynomial in $t$, which in general, has complex coefficients, and which, when $u$ and $v$ denote polynomials in $t$ with real coefficients, may be written in the form

$$
\Psi(t)=u+i v .
$$


$u$ and $v$ must not vanish identically simultaneously, and may reduce to constants. Now

$$
(r-i s)(u+i v)=r u+s v+i(r v-s u),
$$

so that from (10)

Hence

$$
\begin{aligned}
& F(t)=r u+s v, \\
& G(t)=r v-s u .
\end{aligned}
$$

Theorem 2: Every rational circular curve may be parametrically represented in the form

$$
\begin{aligned}
& x=\frac{r u+s v}{\left(r^{2}+s^{2}\right) \Phi}, \\
& y=\frac{r v-s u}{\left(r^{2}+s^{2}\right) \Phi},
\end{aligned}
$$

where all letters on the right hand side stand for polynomials in $t$, such that $r$ and $s$ have no common roots. The polynomials $r u+s v, r v-s u,\left(r^{2}+s^{2}\right) \Phi$, likewise have no common roots. When the degree of $r^{2}+s^{2}$ is $2 k$, then the curve has each of the circular points as a k-fold point. Conversely, from (12) follows readily that every parametric representation as defined by (12) represents a circular curve with the circular points as $k$-fold points.

When $\Phi$ reduces to a constant, which we may place equal to 1 , and the degrees of $r u+s v$ and $r v-s u$ are equal to or less than that of $r^{2}+s^{2}$, all infinite points of the curve are at the circular points, so that, in this case, the curve is isotropic.

\section{Isotropic Curves in a Complex Plane.}

From the parametric representation of an isotropic curve

$$
x^{\prime}=\frac{r u+s v}{r^{2}+s^{2}}, \quad y^{\prime}=\frac{r v-s u}{r^{2}+s^{2}},
$$

we find

$$
x^{\prime}+i y^{\prime}=\frac{u+i v}{r+i s} .
$$

If we replace $t$ in $u, v, r, s$ by the complex variable

$$
t=z=x+i y,
$$


and put $x^{\prime}+i y^{\prime}=z^{\prime}$, (14) assumes the form

$$
z^{\prime}=f(z) / g(z)
$$

in which $f(z)=u+i v, g(z)=r+i s$, and $f(z)$ is a polynomial whose degree is at most equal to that of $g(z)$, and has no factor in common with $g(z)$. Moreover $g(z)$ has no real roots. Hence

Theorem 3: By the rational transformation

$$
z^{\prime}=f(z) / g(z)
$$

in which $f(z)$ and $g(z)$ are polynomials as defined above, the real axis of the z-plane is transformed into a rational isotropic curve. On the other hand, when $f(z)$ and $g(z)$ are arbitrarily given in advance, subject to the conditions that the degree of $f(z)$ is at most equal to that of $g(z)$, that $g(z)=0$ has no real roots, and $f(z)=0$ and $g(z)=0$ have no common roots, then (15) always defines an isotropic curve.

The proof of the second part of the theorem follows easily by deriving equations (14) and (13) from (15) in the reversed order.

Instead of separating in $f(z)$ and $g(z)$ the terms with real coefficients from those with complex coefficients, as understood in (14), we can also separate all real from all imaginary terms, when considering $f(z)$ and $g(z)$ as polynomials in $x$ and $y$. Thus, from (15) we get an expression for $z^{\prime}$ of the same form as (14), but in which $u, v, r, s$ are now real polynomials in $x$ and $y$, which satisfy the Riemann-Cauchy differential equations. Formulas (13), with the new meaning of $u$, $v$, $r, s$, represent the same isotropic curve as before, if we let $y=0$, and if we let $x=t$ assume all values of the real axis.

When in (15) $z$ describes the real axis, the cartesian equation of the corresponding isotropic curve described by $z^{\prime}$ is obtained in the following manner: For the real axis $z=\bar{z}$; consequently, when

$$
\begin{aligned}
& f(z)=z^{m}+a_{1} z^{m-1}+a_{2} z^{m-2}+\cdots+a_{m}, \\
& g(z)=b_{0} z^{n}+b_{1} z^{n-1}+b_{2} z^{n-2}+\cdots+b_{n},
\end{aligned}
$$

with $m \leqq n$, we have the conditional equations,

$$
\begin{gathered}
z^{\prime}\left(b_{0} z^{n}+b_{1} z^{n-1}+b_{2} z^{n-2}+\cdots+b_{n}\right) \\
-\left(z^{m}+a_{1} z^{m-1}+a_{2} z^{m-2}+\cdots+a_{m}\right)=0 \\
\bar{z}^{\prime}\left(\bar{b}_{0} z^{n}+\bar{b}_{1} z^{n-1}+\bar{b}_{2} z^{n-2}+\cdots+\bar{b}_{n}\right) \\
-\left(z^{m}+\bar{a}_{1} z^{m-1}+\bar{a}_{2} z^{m-2}+\cdots+\bar{a}_{m}\right)=0 .
\end{gathered}
$$


The resultant of these two equations in $z$ is a polynomial in $z^{\prime}$ and $\bar{z}^{\prime}$, which, when set equal to zero, and on replacing $z^{\prime}$ by $x^{\prime}+i y^{\prime}, \bar{z}^{\prime}$ by $x^{\prime}-i y^{\prime}$, reduces to the required equation of the isotropic curve. As both equations in (16) are of degree $n$, the degree of this curve will, in general, be $2 n$, which is also apparent from (13). This method of finding the curve described by $z^{\prime}$, when $z$ describes the real axis, is valid for any rational transformation between $z$ and $z^{\prime}$, and includes the generation of all rational circular curves.

\section{Rational Circular Cubics.}

As an example for this method, the cartesian equation of all rational circular cubics, with the origin as a singular point, will be derived from the corresponding rational transformation of the superposed complex plane

$$
z^{\prime}=\frac{z^{2}+a z+b}{(\alpha+i \beta) z+(\gamma+i \delta)},
$$

where $a, b, \alpha, \beta, \gamma, \delta$ are real, and $\alpha \delta-\beta \gamma \neq 0$. Equations (16) have now the form

$$
\begin{aligned}
& z^{2}+\left[a-(\alpha+i \beta) z^{\prime}\right] z+b-(\gamma+i \delta) z^{\prime}=0 \\
& z^{2}+\left[a-(\alpha-i \beta) \bar{z}^{\prime}\right] z+b-(\gamma-i \delta) \bar{z}^{\prime}=0 .
\end{aligned}
$$

Eliminating $z$ between (18) and (19), the resulting equation in the cartesian plane reduces to

$$
\begin{aligned}
(\alpha \delta-\beta \gamma)\left(x^{\prime 2}+y^{\prime 2}\right)\left(\beta x^{\prime}+\alpha y^{\prime}\right)+\left(\delta^{2}-a \beta \delta+b \beta^{2}\right) x^{\prime 2} & \\
+(2 \gamma \delta-a \alpha \delta-a \beta \gamma & +2 b \alpha \beta) x^{\prime} y^{\prime} \\
& +\left(\delta^{2}-a \alpha \gamma+b \alpha^{2}\right) y^{\prime 2}=0
\end{aligned}
$$

This may represent any rational circular cubic, with the singular point at the origin, by choosing $a, b, \alpha, \beta, \gamma, \delta$ properly.

\section{Rational Bicircular Quartics.}

Instead of treating quartics in the same manner as circular cubics, the reversed order will here be followed. The problem now is, to find the rational transformation in the complex plane, when the equation of the curve is given. The equation of any bicircular rational quartic, with the origin as a singular 
point, may be written in the form

$$
\begin{aligned}
\left(x^{\prime 2}+y^{\prime 2}\right)^{2}+\left(\alpha x^{\prime}+\beta y^{\prime}\right)\left(x^{\prime 2}+y^{\prime 2}\right) & \\
& -\left(p^{2}+\alpha p\right) x^{\prime 2}+b x^{\prime} y^{\prime}+c y^{\prime 2}=0,
\end{aligned}
$$

in which $p$ is the distance from the origin of one of the four points of intersection of the quartic with the $x^{\prime}$-axis.

The circle

$$
x^{\prime 2}+y^{\prime 2}-p x^{\prime}-2 t y^{\prime}=0,
$$

through this point and the origin, cuts the quartic in only one variable point, corresponding to the parameter $t$. The coordinates of (21) can therefore be expressed rationally in terms of $t$, by solving (21) and (22) simultaneously. The result is

(23) $x^{\prime}=\frac{p\left(4 t^{2}+2 \beta t+c\right)^{2}-2 t(4 p t+2 \alpha t+\beta p+b)\left(4 t^{2}+2 \beta t+c\right)}{\left(4 t^{2}+2 \beta t+c\right)^{2}+(4 p t+2 \alpha t+\beta p+b)^{2}}$,

$$
y^{\prime}=\frac{2 t(4 p t+2 \alpha t+\beta p+b)^{2}}{\left(4 t^{2}+2 \beta t+c\right)^{2}+(4 p t+2 \alpha t+\beta p+b)^{2}} .
$$

If we put $4 t^{2}+2 \beta t+c=r, 4 p t+2 \alpha t+\beta p+b=s$, (23) and (24) may be written in the form

$$
\begin{aligned}
& x^{\prime}=\frac{r u}{r^{2}+s^{2}}, \\
& y^{\prime}=\frac{-s u}{r^{2}+s^{2}},
\end{aligned}
$$

which is in agreement with the general result contained in (13), in which $v=0$. Hence

$$
z^{\prime}=\frac{u}{r+i s}
$$

or, putting $t=z$,

$$
z^{\prime}=\frac{-4(\alpha+p) z^{2}-2 b z+c p}{4 z^{2}+[2 \beta+i(4 p+2 \alpha)] z+i(\beta p+b)}
$$

is the required transformation, which transforms the real axis into the given rational bicircular quartic. 
For example, Bernoulli's lemniscate

$$
\left(x^{\prime 2}+y^{\prime 2}\right)^{2}-2 a^{2}\left(x^{2}-y^{\prime 2}\right)=0
$$

is obtained by the transformation

$$
z^{\prime}=\frac{a \sqrt{2}\left(z^{2}-1\right)}{-z^{2}-2 i z-1} .
$$

6. Transformation of a General Algebraic Curve in the z-Plane.

Instead of restricting ourselves to the rational transformation of the real axis in the z-plane, we may ask the question, what effect the transformation (13) has upon an algebraic curve $C$ of the order $\mu$ and of deficiency $p$, when $u, v, r, s$ are obtained as polynomials of $x$ and $y$ from the rational transformation (15), in which the degree of $g(z)$ is $n$, that of $f(z) m$, with $m \leqq n$. When $C$ does not pass through the common intersections of the curves $r=0$ and $s=0, C$ will intersect the curve $r^{2}+s^{2}=0$ in $2 \mu n$ imaginary points which by (13) are transformed into the circular points at infinity. Each circular point is therefore a $\mu n$-fold point of the transformed curve $C^{\prime}$. Now to a straight line $l^{\prime}$ in the $z^{\prime}$-plane corresponds in the $z$-plane a curve $l$ of order $2 n$. This curve $l$ cuts $C$ in $2 \mu n$ points, which, conversely, are transformed into the $2 \mu n$ intersections of $l^{\prime}$ with $C^{\prime}$. The order of $C^{\prime}$ is therefore $2 \mu n$, and as the circular points absorb all $2 \mu n$ infinite points of $C^{\prime}$, this curve will be an isotropic curve. According to Clebsch,* the deficiency of $C^{\prime}$ is the same as that of $C$, so that a rational curve $C$ is transformed into a rational isotropic curve $C^{\prime}$. Evidently nothing in the generality of representation of rational circular and isotropic curves is lost by taking in place of a general curve $C$, the real axis. It is therefore not necessary, for our purpose, and in this place, to give further details of the relation between $C$ and $C^{\prime}$.

* Vorlesungen über Geometrie, vol. 1, pp. 661-674 (1876). 